\title{
Pim-3 promotes human pancreatic cancer growth by regulating tumor vasculogenesis
}

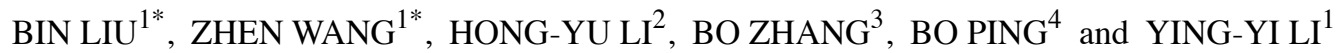 \\ ${ }^{1}$ Cancer Research Institute, Fudan University Shanghai Cancer Center, Department of Oncology, Shanghai Medical College, \\ Fudan University, Shanghai; ${ }^{2}$ Department of Gastroenterology, Shenyang General Hospital, Shenyang, Liaoning; \\ Departments of ${ }^{3}$ Pancreas and Hepatobiliary Surgery, and ${ }^{4}$ Pathology, Fudan University Shanghai Cancer \\ Center, Department of Oncology, Shanghai Medical College, Fudan University, Shanghai, P.R. China
}

Received January 22, 2014; Accepted April 8, 2014

DOI: $10.3892 /$ or.2014.3158

\begin{abstract}
Pim-3, a proto-oncogene with serine/threonine kinase activity, is aberrantly expressed in malignant lesions, but not in normal pancreatic tissues. To assess the role of Pim-3 in human pancreatic carcinogenesis in vivo and to determine the underlying Pim-3 signaling regulatory mechanisms, we established MiaPaca-2 cells overexpressing wild-type Pim-3 or Pim-3 kinase dead mutants (K69M-Pim-3) as well as PCI55 cells stably expressing Pim-3 shRNA or scrambled shRNA in a tetracycline-inducible manner. In addition, we conducted studies utilizing a nude mouse tumor xenograft model. Our results demonstrated that cells stably overexpressing wildtype Pim-3 exhibited functionally enhanced phosphorylation of Bad at $\mathrm{Ser}^{112}$ and increased proliferation. In contrast, the stable inactivation of Pim-3 by K69M-Pim-3 or silencing of Pim-3 expression by Pim-3 shRNA resulted in functionally decreased phosphorylation of Bad at $\mathrm{Ser}^{112}$ and higher apoptotic cells. Following subcutaneous injection of these stable cell lines, nude mice injected with Pim-3 overexpressing cells developed $100 \%$ subcutaneous tumors, together with increased PCNA-positive cells and enhanced intratumoral CD31-positive vascular areas. On the other hand, intratumoral neovascularization and tumor cell proliferation was attenuated in mice injected with Pim-3 kinase inactive cells, eventually reducing tumorigenicity in these mice to $46.6 \%$. Moreover, Pim-3 overexpression upregulated the intratumoral levels of pSTAT3 ${ }^{\text {Try705 }}$, pSurvivin ${ }^{\text {Thr34 }}$, HGF, EGF, FGF-2 and VEGF, while the increases were markedly diminished on Pim-3 kinase inactivation. Collectively, the Pim-3 kinase emerges as being involved in accelerating human pancreatic cancer
\end{abstract}

Correspondence to: Professor Ying-Yi Li, Cancer Research Institute, Fudan University Shanghai Cancer Center, Room 1216, 2nd Building, 270 Dong An Road, Shanghai 200032, P.R. China

E-mail: liyingyi@fudan.edu.cn

*Contributed equally

Key words: Pim-3, pancreatic cancer, survival, angiogenesis development and in promoting tumor neovascularization and subsequent tumor growth. Targeting Pim-3 may play a dual role in halting tumor progression, by promoting tumor cell death and blocking angiogenesis.

\section{Introduction}

Pancreatic cancer is an aggressive malignancy, the fourth leading cause of cancer-related mortality in the United States, with $\sim 44,980$ new diagnoses and $\sim 38,460$ deaths predicted in 2013 (1). Owing to pancreatic cancer characteristics, $>80 \%$ of patients are diagnosed at an advanced stage, thereby losing the probability of surgical resection (2). Despite recent progress in chemotherapy, radiation therapy, and surgical resections, the overall survival rate of pancreatic cancer is still $<5 \%$ (3), with a median survival between 3 and 6 months (4,5). Thus, molecular targeted therapy may be a suitable therapeutic option for human pancreatic cancer treatment.

Pancreatic tumors usually display a ductal, an acinar or an endocrine differentiation. Eighty percent of all pancreatic carcinomas are estimated as pancreatic ductal adenocarcinoma (PDAC) (6). The progression model of PDAC is associated with multiple genetic and epigenetic alterations that result in the deregulation of key proto-oncogenes, tumor-suppressor genes, and signaling pathways, including K-ras, p16, p53, BRCA2, Smad4, EGF/EGFR, c-MET/HGF pathway, Ras/Raf/MAPK pathway, PTEN/PI3K/AKT pathway, JAK/STAT pathway and Wnt signaling. However, roles of other proto-oncogenes and tumor suppressor genes in PDAC development remain elusive (7).

We previously identified Pim-3, a proto-oncogene with serine/threonine kinase activity, as the gene selectively expressed in human pancreatic cancer tissues, but not in the normal pancreas (8). Pim-3 was originally identified as a depolarization-induced gene, KID-1, in PC12 cells, a rat pheochromocytoma cell line (9). Subsequently, Deneen et al (10) demonstrated that Pim-3 gene transcription was enhanced in EWS/ETS-induced malignant transformation of NIH3T3 cells, suggesting the involvement of Pim-3 in tumorigenesis. Consistently, we observed that Pim-3 expression was enhanced in malignant lesions, but not in normal tissues of human endoderm-derived organs, including the pancreas (8), liver 
(11), colon (12) and stomach (13). Moreover, hepatocellular carcinoma development was accelerated in mice selectively expressing the Pim-3 transgene in the liver, when these mice were treated with a hepatocarcinogen (14). Furthermore, Pim-3 can inactivate a proapoptotic molecule, $\mathrm{Bad}$, and maintain the expression of an antiapoptotic molecule, $\mathrm{Bcl}-\mathrm{X}_{\mathrm{L}}$, and prevent apoptosis of human pancreatic cancer and colon cancer cells $(8,12)$. In addition, we demonstrated that TCTP-mediated enhancement in Pim-3 protein stability can be involved in pancreatic carcinogenesis (15). Thus, Pim-3 is a key player in pancreatic tumorigenesis.

However, regulatory mechanisms of Pim-3 signaling networks in vivo are not well understood. In the present study, we discovered that the incidence of human pancreatic cancer was significantly decreased from $100 \%$ to $46.6 \%$ in nude mice subcutaneously injected with cells stably expressing the inactive Pim-3 kinase (K69M-Pim-3) compared with mice injected with cells overexpressing wild-type Pim-3. Moreover, Pim-3 kinase inactivation reduced the expression of several angiogenesis factors, such as HGF, EGF, FGF-2, as well as VEGF, and subsequently prevented neovascularization of nude mice xenografts. These results suggested that Pim-3 kinase activity played a crucial role in accelerating human pancreatic cancer development and in promoting tumor neovascularization and subsequent tumor growth.

\section{Materials and methods}

Cell culture and reagents. Human pancreatic cancer cell lines, MiaPaca-2 (16) and PCI55 (17), were maintained in RPMI1640 (BioWest, Nuaillé, France) containing 10\% fetal bovine serum (FBS; BioWest). Human embryonic kidney HEK293T cells were maintained in DMEM (Sigma) containing 5\% FBS. All cells were cultured in $5 \% \mathrm{CO}_{2}$ at $37^{\circ} \mathrm{C}$. The following monoclonal antibodies (mAbs) and polyclonal antibodies (pAbs) were commercially obtained: rabbit anti-Pim-3 mAbs, rabbit anti-phospho-Ser ${ }^{12}$ Bad pAbs, rabbit anti-phospho-Ser ${ }^{136} \mathrm{Bad}$ pAbs, rabbit anti-phospho-Ser ${ }^{155}$ Bad pAbs, rabbit anti-STAT3 mAbs, rabbit anti-survivin mAbs, rabbit anti-phospho-Ser ${ }^{34}$ survivin mAbs, mouse anti-phospho-try705 STAT3 mAbs, and mouse anti-PCNA mAbs (Cell Signaling Technology, Beverly, MA, USA); rabbit anti- $\beta$-actin mAb (Sigma-Aldrich, St. Louis, MO, USA); mouse anti-Bad mAb and goat antimouse HRP-IgG pAbs (Santa Cruz Biotechnology, Santa Cruz, CA, USA); goat anti-rabbit HRP-IgG pAbs (Pierce Biotechnology, Rockford, IL, USA); rabbit anti-CD31 mAbs (Abcam, Cambridge, MA, USA).

Retroviral vector construction and retrovirus production. Full-length wild-type human Pim-3 cDNA and kinase-dead mutant human Pim-3 (K69M) cDNA were inserted into the PmaCI and HpaI sites of the pMEI-5 Neo retroviral expression vector. Retrovirus was produced by transfecting $293 \mathrm{~T}$ cells with retroviral vectors using the Retrovirus Packaging Kit Ampho (Takara, Dalian, China), according to the manufacturer's instructions. Two days post-transfection, the virus-containing supernatant was collected, passed through $0.45-\mu \mathrm{m}$ syringe filters, and the virus titer was determined using the Retrovirus Titer Set (Takara) according to the manufacturer's instructions.
Establishment of Pim-3 and K69M-Pim-3 stable cell lines. Human pancreatic cancer cells, MiaPaca-2, as target cells were incubated with the retroviral supernatant for $24 \mathrm{~h}$. Subsequently, the infected MiaPaca-2 cells were cultured in $10 \%$ FBS-containing RPMI-1640 in the presence of $800 \mu \mathrm{g} / \mathrm{ml}$ G418 (Gibco) for 2-3 weeks. The surviving cells were isolated using cloning rings and analyzed for Pim-3 or K69M-Pim-3 expression using western blotting. Cells with the highest expression levels of Pim-3 and K69M-Pim-3 were designated as MiaPaca-2-Pim-3 cells and MiaPaca-2-Pim-3K69M cells, respectively, and used for subsequent experiments.

Construction of tetracycline-inducible Pim-3 shRNA expression vectors. pSingle-tTS-Pim-3shRNA and pSingletTS-scramble shRNA expression constructs were provided by Professor Naofumi Mukaida (Cancer Research Institute, Kanazawa University, Japan). In brief, the selected short interfering RNA target sequence in Pim-3 (5'-GCACGUGGUG AAGGAGCGG-3' corresponding to 642-661 residues) and non-specific control short interfering RNA duplexes (5'-GCG CGCUUUGUAGGAUUCG-3') were designed as previously described (8), while small hairpin RNA (shRNA) encoding oligonucleotides were synthesized by Ambion (Austin, TX, USA). The annealed shRNA was inserted into the HindIII and XhoI sites of the pSingle-tTS-shRNA vector (Clontech Laboratories-Takara Bio Inc., Japan) and designated as pSingle-tTS-Pim-3shRNA and pSingle-tTS-scramble shRNA, respectively. The pSingle-tTS-shRNA vector expresses the tetracycline-controlled transcriptional suppressor (tTS), which, in turn, controls the expression of Pim-3 shRNA or scramble shRNA inserted into the shRNA cloning site.

Transfection of shRNA expression vectors and generation of stable cell lines. PCI55 cells were transfected with pSingle-tTS-Pim-3shRNA or pSingle-tTS-scramble shRNA, which functioned as the control, using Lipofectamine 2000 (Invitrogen, Carlsbad, CA, USA) according to the manufacturer's protocol. The transfected PCI55 cells were cultured in $10 \%$ FBS-containing RPMI-1640 in the presence of $800 \mu \mathrm{g} / \mathrm{ml}$ G418 (Gibco) for 2-3 weeks and monoclonal cells were isolated. Thirty colonies were expanded and screened for inducible expression using media supplemented with or without $1 \mu \mathrm{g} / \mathrm{ml}$ tetracycline for $48 \mathrm{~h}$. Cells were analyzed for Pim-3 expression using western blotting. The stably transfected Pim-3 shRNA cells with markedly diminished Pim-3 protein expression under inducible conditions were designated as PCI55-Pim3shRNA cells, while the stably transfected scramble shRNA cells maintaining Pim-3 protein expression under inducible conditions were designated as PCI55-Scramble shRNA cells. These two stable cell lines were used for subsequent experiments.

Western blotting. Cells $\left(2 \times 10^{6}\right)$ were harvested and rinsed twice with PBS. Cell extracts were prepared with lysis buffer [20 mM Tris ( $\mathrm{pH} 7.5), 0.1 \%$ Triton $\mathrm{X}-100,0.5 \%$ deoxycholate, $1 \mathrm{mM}$ phenylmethylsulfonyl fluoride, $10 \mu \mathrm{g} / \mathrm{ml}$ aprotinin, and $10 \mathrm{~g} / \mathrm{ml}$ leupeptin] and cleared by centrifugation at $10,000 \mathrm{x} \mathrm{g}$, $4^{\circ} \mathrm{C}$ for $15 \mathrm{~min}$. Total protein concentration was measured using the BCA assay kit (Sigma) with BSA as a standard, according to the manufacturer's instructions. Cell extracts containing 
$30 \mu \mathrm{g}$ of total protein were subjected to $10 \%$ SDS-PAGE, and the resolved proteins were transferred electrophoretically to polyvinylidene difluoride membranes (Millipore). Equal protein loading was confirmed by Coomassie blue (Bio-Rad Laboratories, Hercules, CA, USA) staining of the gel. After blocking with TBST containing $0.2 \%$ BSA for $1 \mathrm{~h}$ at room temperature, membranes were incubated with $3-5 \mu \mathrm{g} / \mathrm{ml}$ antibodies in PBS containing $0.1 \%$ Tween- 20 overnight at $4^{\circ} \mathrm{C}$, followed by incubation with ImmunoPure peroxidase-conjugated anti-rabbit IgG or anti-mouse IgG. Chemiluminescent detection (Thermo Scientific Pierce, Rockford, IL, USA) was performed in accordance with the manufacturer's instructions. The blotted membrane was then treated with the SuperSignal West Dura Extended Duration Substrate and signals were detected using the LAS-4000 mini CCD camera. Blots were performed at least three times in independent experiments. For some experiments, tumor tissues were prepared with RIPA lysis buffer.

Cell viability assay. Cell viability was determined by the Cell Proliferation Assay and Cytotoxicity Assay kit (CCK-8; Dojindo Laboratories, Kumamoto, Japan), according to the manufacturer's instructions. Logarithmically growing cells were plated at $2 \times 10^{3}$ cells/well in 96 -well culture plates (Corning), and allowed to adhere overnight. This time-point was designated as day 0 . The cell viability was determined every day by adding $10 \mu \mathrm{l}$ of CCK-8 reagent to each well. After incubation at $37^{\circ} \mathrm{C}$ for $2 \mathrm{~h}$, the absorbance at $450 \mathrm{~nm}$ was measured and ratios of cell numbers were determined by comparison of the number of cells at day 0 . Each independent experiment was performed three times.

Cell apoptosis analysis. The cells were trypsinized and $2 \times 10^{5}$ cells were plated in a 6-well plate. After incubation at $37^{\circ} \mathrm{C}$ for $24 \mathrm{~h}$, cells were washed and resuspended in $0.5 \mathrm{ml}$ of PBS, $5 \mu \mathrm{l}$ Annexin V-FITC (Invitrogen), and $1 \mu \mathrm{l}$ propidium iodide $(100 \mu \mathrm{g} / \mathrm{ml}$; Invitrogen). The cells were incubated for $30 \mathrm{~min}$ on ice and then analyzed by flow cytometry (Cytomics ${ }^{\mathrm{TM}}$ FC 500; Beckman Coulter, Miami, USA) for each treatment. The apoptotic fraction was estimated by dividing the number of apoptotic cells by the total number of cells (minimum of $10^{4}$ cells). Data were analyzed using Cytomics FC 500 with CXP Software (Beckman Coulter). All observations were reproduced at least three times in independent experiments.

Xenograft mouse model. Female Balb/c nude mice (6-8 weeks of age, weighing 18-20 g, and specific pathogen free) were obtained from Shanghai SLAC Laboratory Animal Co. (Shanghai, China). Before the experiment, mice were divided into six groups (MiaPaca-2, MiaPaca-2-Pim-3, MiaPaca-2Pim-3K69M, PCI55, PCI55-Pim-3shRNA and PCI55-scramble shRNA) according to body weight, and each cell line ( $4 \times 10^{6} /$ site $)$ was injected subcutaneously into the right flank of the nude mice. After establishment of the nude mice xenograft model, tumor dimensions were measured every 3-4 days using micrometer calipers. Tumor volumes were calculated using the following formula: Volume $=1 / 2 \mathrm{ax} \mathrm{b}^{2}$, where $\mathrm{a}$ and $\mathrm{b}$ represent the larger and smaller tumor diameters, respectively. At 30 days after the tumor cell injection, tumor tissues were removed and subjected to immunohistochemical analysis. All animal experi- ments were performed in compliance with the Guideline for the Care and Use of Laboratory Animals of Fudan University. The protocol was approved by the Committee on the Ethics of Animal Experiments of Fudan University (Permit Number, SYXK(Hu)2009-0082).

Immunohistochemical analysis. Paraffin-embedded tissue sections were deparaffinized in xylene and rehydrated through graded concentrations of ethanol (70-100\%). Following incubation with $0.3 \%$ hydrogen peroxide, sections were incubated with 3\% normal goat serum (DakoCytomation, Glostrup, Denmark). Subsequently, the slides were treated with rabbit anti-PCNA IgG $(3 \mu \mathrm{g} / \mathrm{ml})$, anti-CD31 $\operatorname{IgG}(3 \mu \mathrm{g} / \mathrm{ml})$, and anti-VEGF $\operatorname{IgG}(3 \mu \mathrm{g} / \mathrm{ml})$, followed by incubation with goat anti-rabbit IgG at room temperature for $1 \mathrm{~h}$. PCNA and CD31 immunoreactivity was visualized by using the Vectastain Elite $\mathrm{ABC}$ kit and the Vectastain DAB substrate kit (Vector Laboratories, Burlingame, CA, USA). The slides were counterstained with ChemMate Hematoxylin (DakoCytomation), mounted and observed under a microscope (BX-50; Olympus, Tokyo, Japan). The PCNA-positive cell numbers in each animal were determined in 10 randomly chosen fields at $x 400$ magnification by an examiner blinded to the experimental procedures. The CD31-positive vascular areas were determined as previously described (18).

Immunofluorescence analysis of apoptotic cells in xenograft specimens. Frozen tumor xenograft specimens were stained using a fluorescent terminal deoxynucleotidyl transferasemediated nick end labeling-based apoptosis detection kit (In Situ Cell Death Kit; Takara), in accordance with the manufacturer's instructions. Fluorescence microscopy was performed using a $\mathrm{x} 40$ objective (Zeiss Plan-Neofluar) on an Olympus Eclipse TE2000-S inverted phase microscope (Olympus, Melville, NY, USA). Images were analyzed using Image-Pro Plus software version 4.0. The apoptosis-positive cell numbers in each animal were determined in 10 randomly chosen fields at $\mathrm{x} 400$ magnification by an examiner blinded to the experimental procedures.

Real-time RT-PCR. Total RNA was extracted using the TRIzol LS reagent (Invitrogen). mRNA was reverse-transcribed using the SuperScript First-Strand Synthesis System (Invitrogen). Real-time PCR was performed using the Applied Biosystems 7900HT PCR system with 2X QuantiFast SYBR-Green PCR Master Mix (Qiagen), $1 \mu \mathrm{M}$ primers (Table I), and $<100 \mathrm{ng}$ cDNA in a $25 \mu 1$ reaction mixture. Relative expression of target genes was analyzed by the $\Delta \Delta \mathrm{Ct}$ method. Results are expressed as means $\pm \mathrm{SD}$.

Statistical analysis. The means \pm SD were calculated for all parameters determined. Statistical significance was evaluated by one-way ANOVA, followed by the Tukey-Kramer test, using SPSS 10 software (IBM, Inc., Chicago, IL, USA). P-values $<0.05$ were considered to indicate a statistically significant result.

\section{Results}

Establishment of overexpression of Pim-3 or K69M-Pim-3 cells and Tet-inducible Pim-3 shRNA or scramble shRNA 
Table I. Primer sequences for quantitative PCR.

Sense (5'-3')

Antisense (5'-3')

\begin{tabular}{lll}
\hline MMP-2 & TACAGGATCATTGGCTACACACC & GGTCACATCGCTCCAGACT \\
MMP-9 & TGTACCGCTATGGTTACACTCG & GGCAGGGACAGTTGCTTCT \\
EGF & TGGATGTGCTTGATAAGCGG & ACCATGTCCTTTCCAGTGTGT \\
FGF-2 & AGAAGAGCGACCCTCACATCA & CGGTTAGCACACACTCCTTTG \\
VEGFA & AGGGCAGAATCATCACGAAGT & AGGGTCTCGATTGGATGGCA \\
PDGFA & GCAAGACCAGGACGGTCATTT & GGCACTTGACACTGCTCGT \\
PDGFB & CTCGATCCGCTCCTTTGATGA & CGTTGGTGCGGTCTATGAG \\
HGF & GCTATCGGGGTAAAGACCTACA & CGTAGCGTACCTCTGGATTGC
\end{tabular}

A
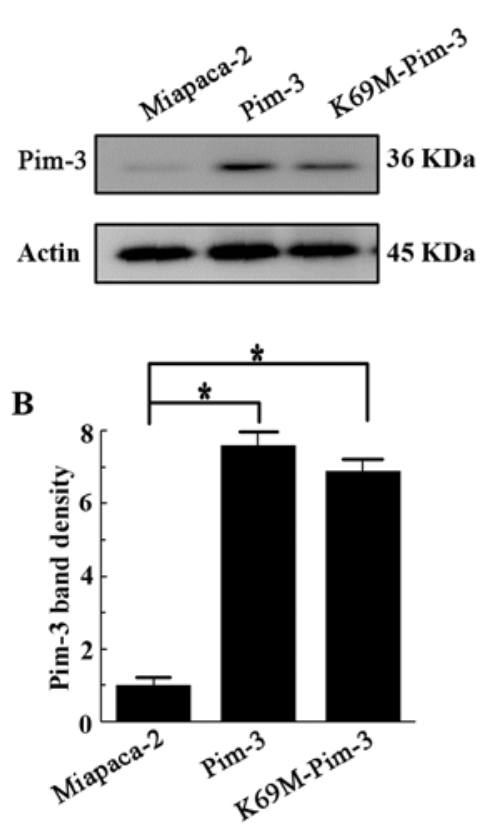

C

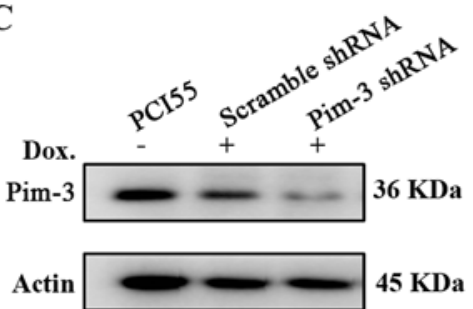

D

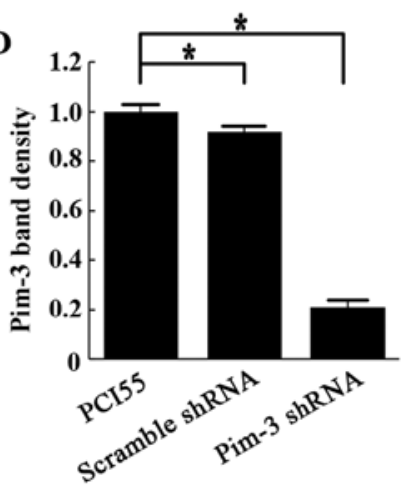

$\mathbf{E}$

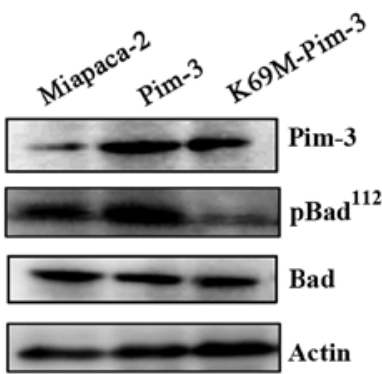

F

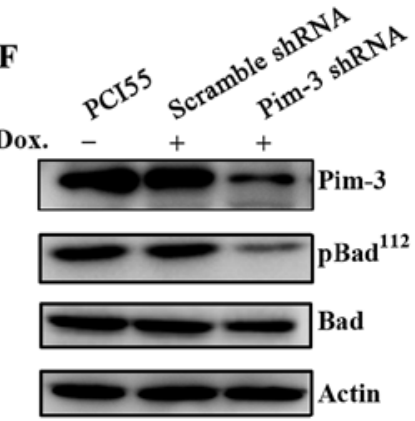

Figure 1. Stable establishment of overexpression of Pim-3 or K69M-Pim-3 cells, and Tet-inducible Pim-3 shRNA or scramble shRNA expressing cells. (A) Overexpression of Pim-3 or K69M-Pim-3 in MiaPaca-2 cells. After G418 selection and isolation of monoclonal cells, cell lysates were obtained from stable cells $48 \mathrm{~h}$ after passage and the resultant lysates were subjected to immunoblotting with Pim-3 antibodies. Representative results from three independent experiments. $\beta$-actin was used to demonstrate equal protein loading. (B) Graphical representation of Pim-3 expression normalized against $\beta$-actin expression. Values in the graph are presented as means $\pm \mathrm{SD}, \mathrm{n}=3$. ${ }^{*} \mathrm{P}<0.05$. (C) Ablation of Pim-3 protein expression using Pim-3 shRNA in PCI55 cells. After monoclonal stable cells were cultured in media supplemented with $1 \mu \mathrm{g} / \mathrm{ml}$ tetracycline for $48 \mathrm{~h}$, cell lysates were obtained and subjected to immunoblotting with Pim-3 antibodies. Representative results from three independent experiments. $\beta$-actin was used to demonstrate equal protein loading. (D) Graphical representation of Pim-3 expression normalized against $\beta$-actin expression. Values in the graph are presented as means $\pm S D, n=3$. " $P<0.05$. (E and F) Analysis of Bad phosphorylation at $\operatorname{Ser}^{12}$ in stable cells. Cells lysates were obtained from stable cells, $48 \mathrm{~h}$ after passage, and resultant lysates were subjected to immunoblotting with the indicated antibodies. Representative results from three independent experiments. $\beta$-actin was used to demonstrate equal protein loading.

expressing cells. To demonstrate the role of Pim-3 in human pancreatic carcinogenesis, we established MiaPaca- 2 cells overexpressing the Pim-3 or K69M-Pim-3 gene using retroviral vectors, which inserted the wild-type human Pim-3 cDNA or kinase-dead mutant (K69M) Pim-3 cDNA. Furthermore, we also established Pim-3 gene silenced cell lines using a Tet-inducible Pim-3 shRNA in PCI55 cells, which express high levels of Pim-3 protein compared with MiaPaca-2 cells, as previously described (8). We confirmed the overexpression of Pim-3 or K69M-Pim-3 protein in MiaPaca-2 cells (Fig. 1A and $\mathrm{B}$ ), and that Pim-3 shRNA markedly diminished Pim-3 protein expression compared to control scramble shRNA under inducible conditions in PCI55 cells (Fig. 1C and D).
Since Pim-3 can phosphorylate a proapoptotic molecule Bad at $\mathrm{Ser}^{112}$, we examined the phosphorylation states of Bad to confirm the functionality of these stable pancreatic cancer cell lines. Bad was constitutively phosphorylated at $\operatorname{Ser}^{12}$ in MiaPaca-2 and PCI55 cell lines. Pim-3 kinase inactivation or silencing of Pim-3 expression diminished the amount of phospho-Ser ${ }^{12}$-Bad, without influencing the expression of total Bad protein (Fig. 1E and F). These observations indicate that overexpression of Pim-3 was functional in terms of its capacity to phosphorylate its substrate Bad, and Pim-3 inactivation by its kinase dead mutant or knockdown of Pim-3 expression by Pim-3 shRNA functionally decreased the amount of phospho$\mathrm{Bad}^{\mathrm{Ser} 12}$. 
A

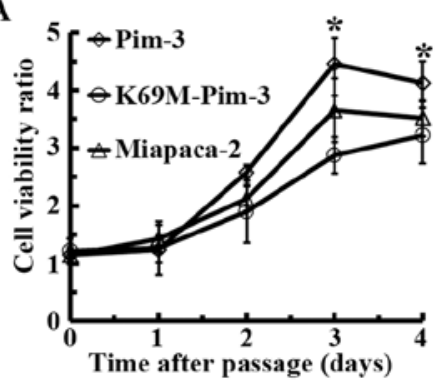

B

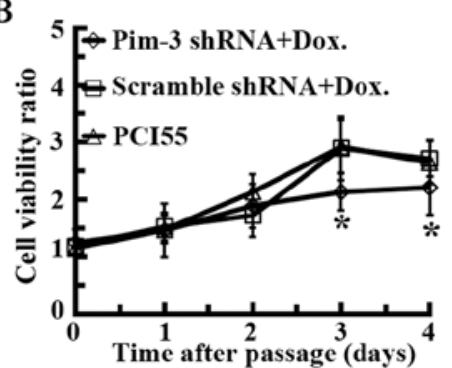

C

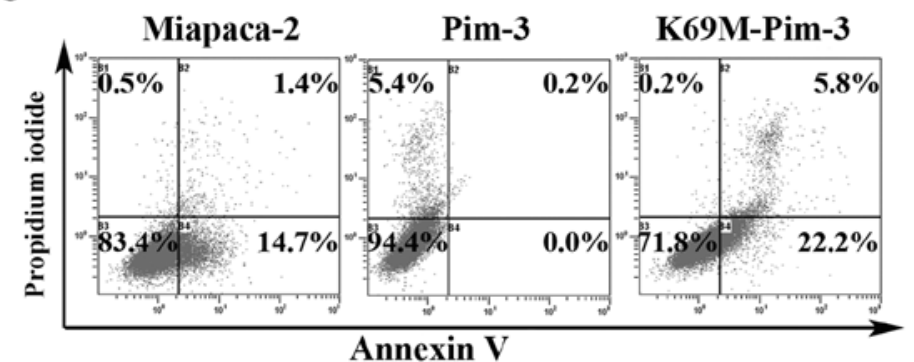

D PCI55 Scramble shRNA Pim-3 shRNA

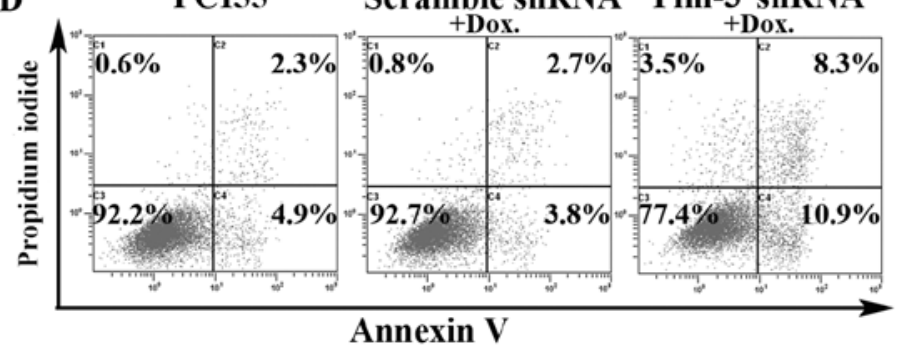

F
$\mathbf{E}$

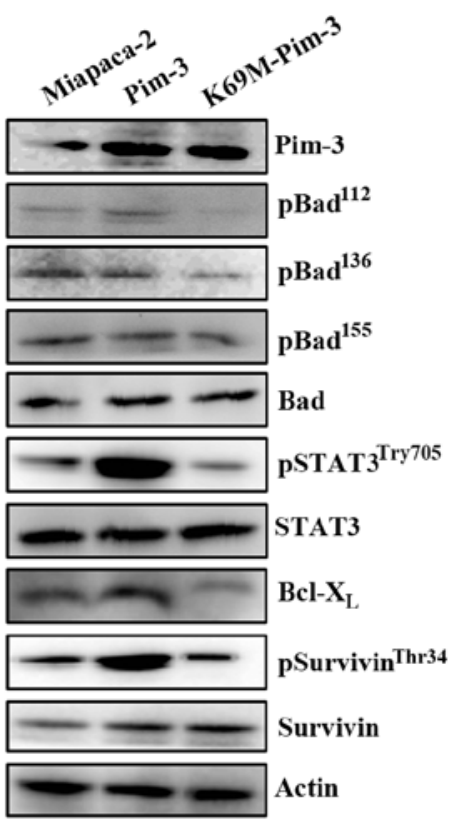

Dox.

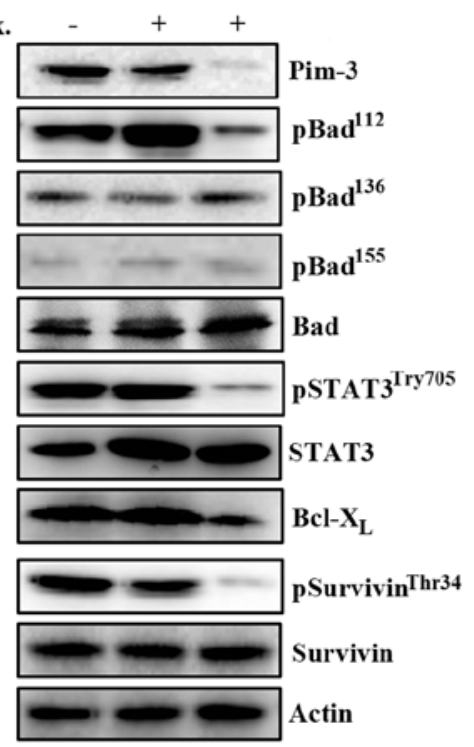

Figure 2. Pim-3 kinase inactivation prevents cell proliferation and promotes apoptosis in pancreatic cancer in vitro. (A and B) The effects of stable overexpression of Pim-3 or K69M-Pim-3 (A), and inducible expression of Pim-3 shRNA or scramble shRNA (B), on cell viability. Cells (1x10 $\left.{ }^{3}\right)$ were seeded in each well of a 96-multiwell plate and cell numbers were determined using the CCK-8 assay, at the indicated times. Relative cell number was determined in comparison with day 0 , and mean \pm SD were calculated and are shown here. Similar experiments were repeated three times and representative results are shown here. ${ }^{*} \mathrm{P}<0.05$. (C and D) The effects of stable overexpression of Pim-3 or K69M-Pim-3 (C), and inducible expression of Pim-3 shRNA or scramble shRNA (D), on cell apoptosis. The cells were harvested $48 \mathrm{~h}$ after passage and subjected to combined staining with Annexin $\mathrm{V}$ and PI. The number in each quadrant indicates the proportion of the cells present in the quadrant. Representative results from three independent experiments are shown here. (E and F) Cells lysates were obtained from cells stably overexpressing Pim-3 or K69M-Pim-3 cells (E), or inducibly expressing Pim-3 shRNA or scramble shRNA (F), 48 h after passage, and the resultant lysates were subjected to immunoblotting with the indicated antibodies. Representative results from three independent experiments are presented. $\beta$-actin was used to demonstrate equal protein loading.

Pim-3 kinase inactivation inhibits cell proliferation and promotes apoptosis in pancreatic cancer cells in vitro. We previously observed that Pim-3 shRNA treatment decreases the in vitro proliferation of various types of cancer cells by enhancing their apoptosis $(8,11,12)$. Consistent with our previous observation, Pim-3 kinase inactivation or silencing of Pim-3 expression decreased the proliferation of human pancreatic cancer cell lines, MiaPaca-2 and PCI55 (Fig. 2A and B), together with an increase in the proportion of both early apoptotic cells (Annexin V-positive and PI-negative) and late apoptotic cells (Annexin V-positive and PI-positive) (Fig. 2C and D), when compared with cells overexpressing Pim-3 or cells stably expressing the scrambled shRNA. To assess the effects of Pim-3 kinase inactivation on the apoptotic process, 
A The development of pancreatic xenograft in nude mice

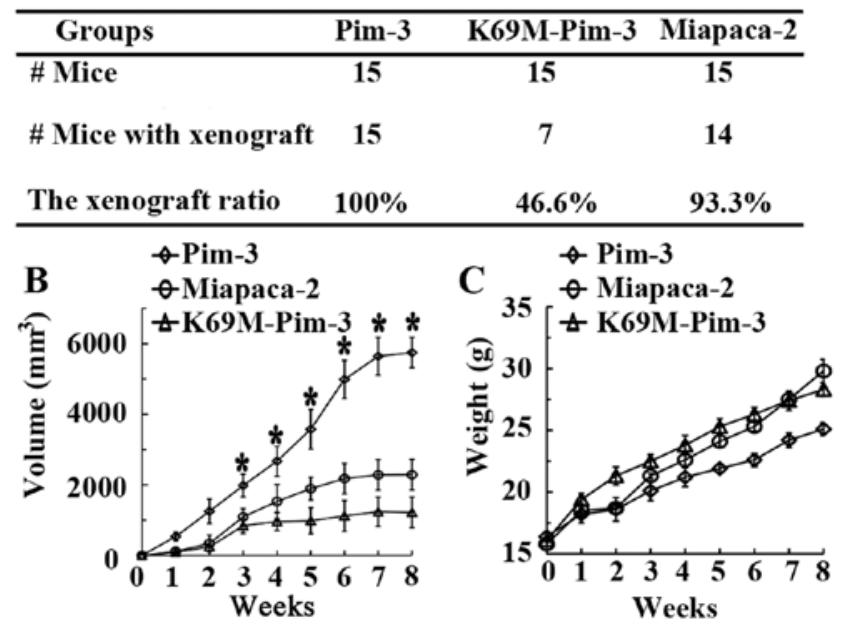

Figure 3. Pim-3 kinase inactivation suppresses tumor growth in nude mice. (A) Incidence of subcutaneous human pancreatic cancer formation with the indicated stable cells. (B) Tumor sizes were measured twice a week. The mean \pm SD were calculated and are shown here $(n=15)$. (C) Body weights were measured twice a week after inoculation. The mean \pm SD were calculated and are shown here $(n=15)$.

we conducted western blotting. As shown in Fig. 2E and F, Bad was constitutively phosphorylated at $\operatorname{Ser}^{112}$ in MiaPaca-2 and PCI55 cell lines; however, Pim-3 kinase inactivation or silencing of Pim-3 expression diminished the amount of phospho-Bad ${ }^{\mathrm{Ser} 112}$, without any effects on the expression of the total Bad protein. Concomitantly, Pim-3 kinase inactivation or silencing of Pim-3 expression decreased $\mathrm{Bcl}-\mathrm{X}_{\mathrm{L}}$ expression, which confirmed our previous results (8). Moreover, compared with Pim-3 overexpression, Pim-3 kinase inactivation decreased phospho-survivin ${ }^{\text {Thr34 }}$ and phospho-STAT3 ${ }^{\text {Try705, }}$, that are an upstream of $\mathrm{Bcl}-\mathrm{X}_{\mathrm{L}}$, with only few effects on total survivin and STAT3. Collectively, these results suggested that the Pim-3 overexpression might increase phosphorylation of Stat3, survivin and Bad, resulting in reduced apoptosis, and eventually promoting human pancreatic carcinogenesis. Inversely, Pim-3 kinase inactivation reverses this effect.

Pim-3 kinase inactivation suppresses pancreatic tumorigenesis in nude mice. Results from preliminary experiments demonstrated that nude mice subcutaneously inoculated with 4 million PCI55 cells failed to develop tumors. Thus, to establish the crucial role of Pim-3 in pancreatic carcinogenesis, we injected the same number of stable Pim-3-MiaPaca-2, K69MPim-3-MiaPaca-2 or parent MiaPaca-2 cells subcutaneously into nude mice. At 30 days following tumor inoculation, we found that $93.3 \%(14 / 15)$ of parental MiaPaca-2 group mice and $100 \%(15 / 15)$ of Pim-3-MiaPaca-2 group mice developed subcutaneous tumors (Fig. 3A). In contrast, only 46.6\% (7/15) of K69M-Pim-3 group mice developed tumors following subcutaneous injection of tumor cells (Fig. 3A). Moreover, mice injected with Pim-3 overexpressing cells exhibited progressive tumor growth compared with parental MiaPaca-2 cells, whereas the growth rate of K69M-Pim-3 tumor cells in nude mice was significantly decreased (Fig. 3B). Throughout the trial period, none of the mice presented with loss in body weight (Fig. 3C). These results indicated that Pim-3 plays a crucial role in subcutaneous pancreatic carcinogenesis in nude mice, and Pim-3 kinase inactivation suppresses tumor growth in vivo.
A

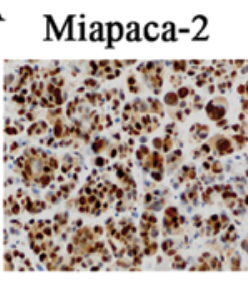

C Miapaca-2

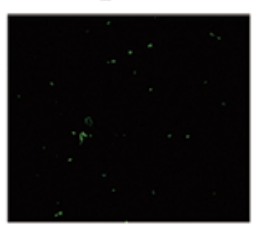

Pim-3

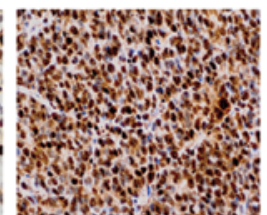

Pim-3

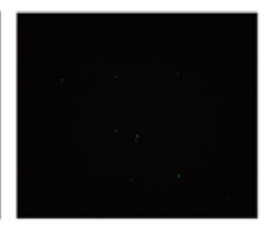

K69M-Pim-3

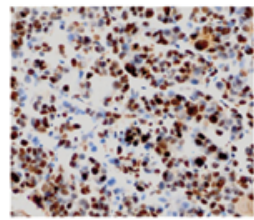

K69M-Pim-3

\section{B}

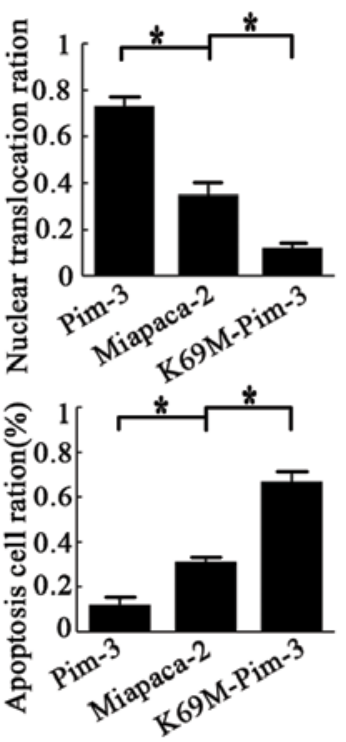

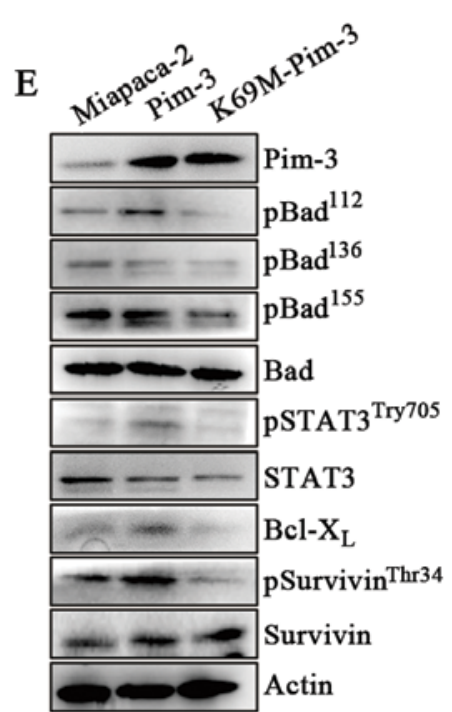

Figure 4. Pim-3 kinase inactivation prevents pancreatic cancer cell proliferation and promotes apoptosis in vivo. (A and B) Tumor tissues were removed at 30 days after tumor injection and subjected to immunostaining with anti-PCNA antibody. (A) Representative results from 5 independent animals are shown, with an original magnification of $x 200$. (B) PCNA-positive cells were determined and are shown ( $\mathrm{n}=5)$. (C and D) Tumor tissues were excised at 30 days after tumor injection and subjected to immunofluorescence analysis for apoptotic cells. (C) Representative results from five independent animals are shown, with an original magnification of x200. (D) TUNEL-positive cells were calculated and are shown ( $\mathrm{n}=5$ ). (E) The cell lysates were obtained from xenograft tumor tissues and resultant lysates were subjected to immunoblotting with the indicated antibodies. Representative results from three independent experiments. $\beta$-actin was used to demonstrate equal protein loading. 
A

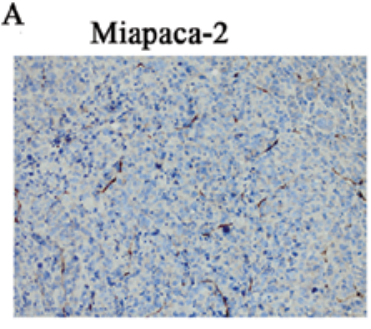

$\mathrm{C}$

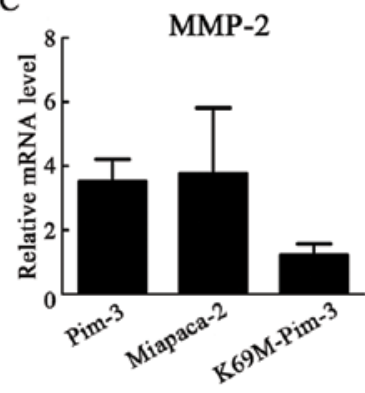

E

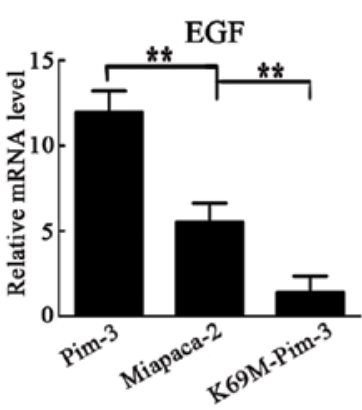

$\mathrm{H}$

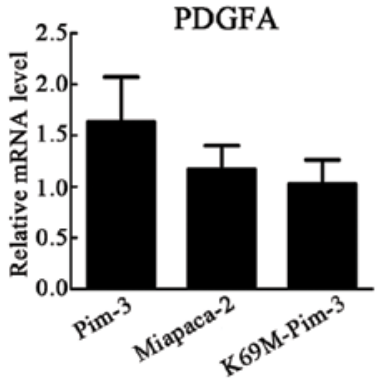

Pim-3

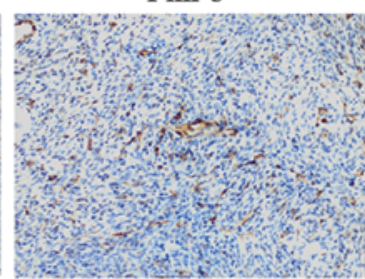

K69M-Pim-3

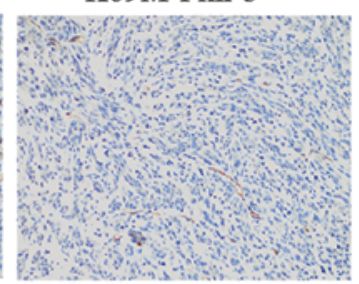

D

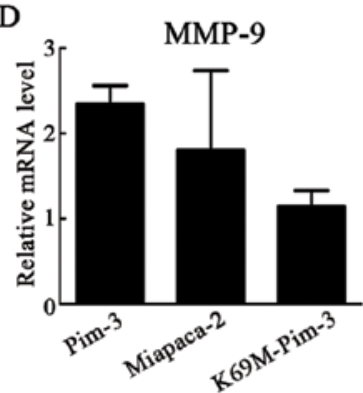

B

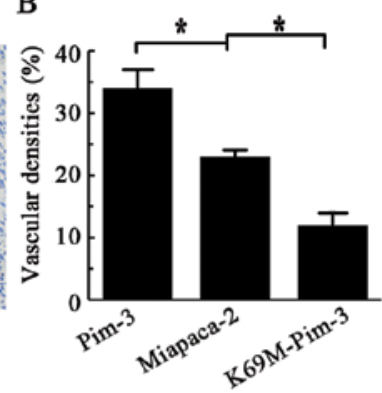

$\mathrm{F}$

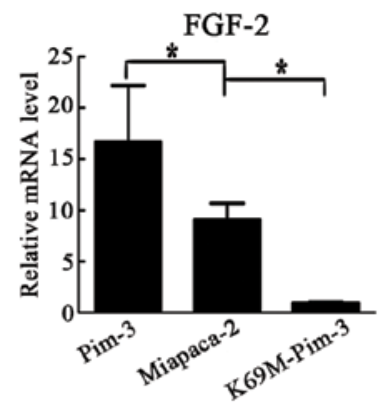

I

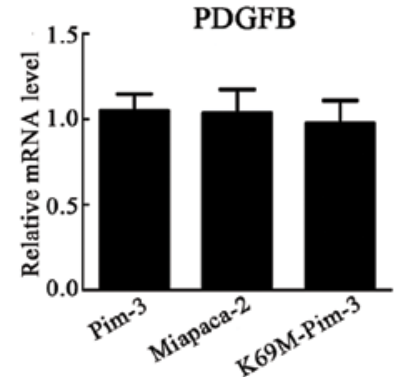

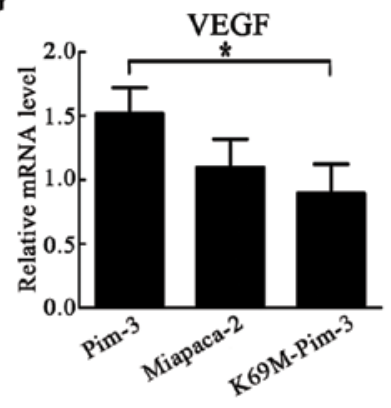

$\mathbf{J}$

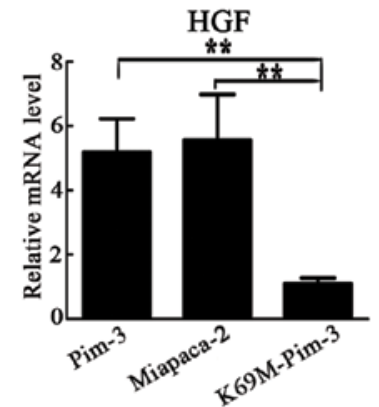

Figure 5. Pim-3 kinase inactivation inhibits neovascularization via inhibition of vasculogenesis in vivo. (A and B) Tumor tissues were removed at 30 days after tumor injection and subjected to immunostaining with the anti-CD31 antibody. (A) Representative results from 5 independent animals, with an original magnification of x200. (B) CD31-positive areas were calculated ( $\mathrm{n}=5$ ). (C-J). Tumor tissues were excised 30 days after tumor injection and total RNAs were obtained. Quantitative RT-PCR was performed on total RNAs to quantify indicated factor mRNA relative to GAPDH mRNA. Each value represents the mean \pm SEM $(n=5)$.

Pim-3 kinase inactivation inhibits proliferation and promotes apoptosis in pancreatic cancer in vivo. We next examined the effects of Pim-3 kinase inactivation on pancreatic cancer cell proliferation and apoptosis in vivo. Histological analysis revealed that Pim-3 kinase inactivation significantly decreased PCNA-positive proliferating cell numbers while increasing TUNEL-positive apoptotic cell numbers (Fig. 4A-D). In contrast, Pim-3 overexpression significantly increased PCNApositive proliferating cell numbers and reciprocally decreased TUNEL-positive apoptotic cell numbers, when compared with the parent MiaPaca-2 cells (Fig. 4A-D). Moreover, in the xenograft tumor tissues, overexpression of Pim-3 increased the amount of phospho-Stat $3^{\text {Try705 }}$, phospho-survivin ${ }^{\text {Thr34 }}$, and phospho-Bad ${ }^{\text {Ser112 }}$, whereas Pim-3 inactivation decreased the expression of these phosphorylated proteins (Fig. 4E). However, the expression of unphosphorylated Stat3, survivin, and Bad proteins were unchanged (Fig. 4E). These observations were consistent with the results of our in vitro experiment.

Reduced neovascularization. Neovascularization is required for tumor growth and accumulating evidence has proved an important role for VEGF in angiogenesis. We recently demonstrated that Pim-3 could promote tumor growth and angiogenesis by stimulating the VEGF pathway (19). Herein, we detected that the intratumoral CD31-positive vascular areas increased following the injection of Pim-3-MiaPaca-2 cells, but significantly decreased following injection of K69M-Pim3-MiaPaca-2 cells (Fig. 5A and B). Hence, we examined VEGF 
mRNA expression together with other angiogenesis factors, including HGF, platelet-derived growth factor (PDGF), fibroblast growth factor (FGF), epithelial growth factor (EGF), matrix metalloproteinases (MMP)-2 and -9. The intratumoral mRNA expression of VEGF, EGF, FGF-2 and HGF, but not MMP-2 and MMP-9 with gelatinase activity was significantly increased in mice injected with Pim-3 overexpressing cells, while their expression was markedly reduced in mice injected with Pim-3 kinase inactive cells, to the parental MiaPaca-2 cells (Fig. 5C-J). These observations may mirror the fact that neovascularization, an essential process for pancreatic carcinogenesis, was augmented in Pim-3 overexpression compared with that observed for Pim-3 kinase inactivation, as demonstrated by increasing in CD31-positive areas in the tumor tissue.

\section{Discussion}

The diagnosis of human pancreatic cancer is often difficult, and in most patients the tumor is already disseminated when discovered. We previously observed that Pim-3, a protooncogene with serine/threonine kinase activity, was aberrantly expressed in cancer cells but not in the normal cells of the pancreas. Moreover, the $\operatorname{Ser}^{112}$ phosphorylation and inactivation of Bad by Pim-3 maintains the expression of Bcl- $\mathrm{X}_{\mathrm{L}}$ and, thus, prevents apoptosis of human pancreatic cancer cells (8). Silencing Pim-3 expression can retard in vitro cell proliferation of pancreatic cancer by promoting apoptosis (8). A recent report demonstrated that Pim-3 suppression can sensitize pancreatic cancer cells to gemcitabine (20). Thus, Pim-3 might be a novel target for the treatment of refractory pancreatic cancer. Since the regulatory mechanisms of Pim-3 signaling networks in vivo are not well understood, we established stable cell lines that overexpress wild-type or the kinase-dead form of Pim-3 (K69M-Pim-3), and utilized a nude mouse tumor xenograft model to assess the regulatory mechanisms of Pim-3 in human pancreatic carcinogenesis in vivo.

Gene delivery in cancer cells can be achieved using transient transfection and stable transfection methods. Stable transfection methods are more appealing and enable continuing expression of the transgene. In the present study, we established overexpression of Pim-3 or K69M-Pim-3, in which Lys-69 in the ATP binding domain was replaced by methionine, rendering its kinase domain non-functional in MiaPaca-2 cells. We also established expression of the Pim-3 shRNA or scramble shRNA in a tetracycline-inducible manner in PCI55 cells, which contain high levels of Pim-3 protein compared with MiaPaca-2 cells, as previously described (8). Kinase activation generally requires a post-translational modification, in particular, phosphorylation in its regulatory domain. However, other members of the Pim kinase family, Pim-1 and Pim-2, are constitutively active without any further alteration in their conformation, as they lack any regulatory domain (21), as does Pim-3 (11). Consistently, stable expression of Pim-3 exhibited enhanced phosphorylation of Bad at $\mathrm{Ser}^{112}$, whereas stable expression of K69M-Pim-3 significantly attenuated phosphorylation of Bad at its Ser $^{112}$ in MiaPaca-2 cells. Moreover, Pim-3 shRNA expression in PCI55 cells, but not in parental PCI55 cells or scramble shRNA-PCI55 cells, markedly reduced the expression of phospho-Bad ${ }^{\text {Ser112. }}$
Thus, Pim-3 inactivation or knockdown of Pim-3 expression by Pim-3 shRNA can functionally decrease the amount of phospho-Bad ${ }^{\mathrm{Ser112}}$. Moreover, Pim-3 kinase inactivation or silencing of Pim-3 expression decreased the cell proliferation of human pancreatic cancer cell lines, MiaPaca-2 and PCI55, together with increasing the apoptotic cells compared with that observed for cells overexpressing Pim-3 or cells expressing the scrambled shRNA.

Deneen et al (10) demonstrated that Pim-3 gene transcription was enhanced in EWS/ETS-induced malignant transformation of NIH3T3 cells, suggesting the involvement of Pim-3 in tumorigenesis. In line with these observations, we demonstrated that the development of hepatocellular carcinoma was accelerated in mice expressing the Pim-3 transgene selectively in the liver, when these mice were treated with a hepatocarcinogen (14). Moreover, forced expression of Pim-3 can promote anchorage-independent growth and co-expression of a kinase-deficient Pim-3 mutant can attenuate EWS/FLI-mediated NIH3T3 tumorigenesis in nude mice (22). Our data demonstrated that overexpression of Pim-3 in the MiaPaca-2 human pancreatic cancer cells developed $100 \%$ $(15 / 15)$ of subcutaneous tumors and exhibited progressive tumor growth, whereas Pim-3 kinase inactivation decreased tumorigenicity to $46.6 \%(7 / 15)$ and inhibited tumor growth. However, subcutaneous inoculation with $4 \times 10^{6}$ PCI55 cells into nude mice did not develop tumors. These observations prompted us to investigate the mechanism of Pim-3 kinase inactivation on decreasing pancreatic carcinogenesis, including cell apoptosis and angiogenesis.

An elevated activity of Stat 3 has been frequently observed in a wide variety of human tumors including pancreatic cancer (23-26). Several lines of evidence demonstrated that the gene expression of Pim-1 and Pim- 2 could be regulated by IL-6-gp130-mediated signal transducers and activators of transcription (STAT) family $(27,28)$. Moreover, Pim-3 expression is enhanced in murine embryonic stem cells by leukemia inhibitory factor/gp130-dependent signaling and Stat3 transcription factor (29). However, transfection of a dominant negative form of Stat 3 failed to inhibit the promoter activity of the Pim-3 gene in human pancreatic cancer cells (30). The excessive activation of Stat 3 can promote anti-apoptotic gene expression, such as $\mathrm{Bcl}-\mathrm{X}_{\mathrm{L}}$ and mc-1, as well as promoting cell proliferation in a variety of tumor cells (31-33). We previously showed that Pim-3 can maintain the expression of $\mathrm{Bcl}-\mathrm{X}_{\mathrm{L}}$, but the mechanism was not clear. Recently, Chang et al (34) demonstrated that the knockdown of Pim-3, but not of Pim-1 or Pim-2, in prostate cancer cell line DU-145 results in a significant downregulation of pStat $3^{\text {Try705}}$, indicating Pim-3 kinase is a positive regulator of Stat 3 signaling. In line with these observations, we detected constitutive Stat 3 and phosphorylated pStat $3^{\text {Try705 }}$ expression in human pancreatic cancer cells. Overexpression of Pim-3 increased the expression levels of phospho-Stat $3^{\text {Try705 }}$ and Bcl- $\mathrm{X}_{\mathrm{L}}$, whereas Pim-3 kinase inactivation or ablation of Pim-3 protein expression significantly reduced the expression levels of $\mathrm{pStat} 3^{\text {Try } 705}$ and $\mathrm{Bcl}-\mathrm{X}_{\mathrm{L}}$, while the expression levels of total Stat 3 remained unchanged. However, the mechanism of apoptosis-related $\mathrm{Bcl}-\mathrm{X}_{\mathrm{L}}$ induction by Stat 3 remains to be elucidated.

Survivin is a member of the 'inhibitor of apoptosis' (IAP) gene family of proteins that is barely detected in normal 
tissues $(35,36)$. However, survivin appears to be selectively expressed in transformed cells and in most human cancers, including pancreatic carcinomas $(31,37)$. It has been previously reported that inhibition of Stat 3 signaling blocked the expression of survivin protein and induced apoptosis in breast cancer cells (38). However, Pim-3 kinase inactivation or ablation of Pim-3 protein expression reduced the phosphorylated levels of STAT3 at Try705, but did not influence the expression of total survivin in human pancreatic cancer cells. Several recent studies reported that the therapeutic modulation of survivin is critically regulated by interaction with prominent cell-signaling pathways, such as HIF-1 $\alpha$, HSP90, PI3K/AKT, mTOR, ERK, tumor suppressor genes (p53, PTEN), oncogenes (Bcl-2, Ras), and a wide range of growth factors (EGFR, VEGF) (39). Thus, it is likely that other pathways in human pancreatic cancer cells regulate the expression of survivin. The suppression of apoptotic cell death by survivin requires phosphorylation at Thr34 (40). Survivin can be phosphorylated by cyclin-dependent kinase-1, cyclic AMP, and protein kinase C as well as AKT (40-42). Similarly, overexpression of Pim-3 increased the levels of phospho-survivin ${ }^{\text {Thr34 }}$, whereas Pim-3 kinase inactivation or ablation of Pim-3 protein expression decreased the levels of phospho-survivin ${ }^{\text {Thr34 }}$, similar to Akt (42).

Angiogenesis is widely recognized as a hallmark of cancer (43), and potent neovascularization that contributes to tumor progression was observed in a variety of aggressive malignant tumors (44). We identified a role for the kinase-dead Pim-3 mutant in reducing the CD31-positive vascular regions in the tumor, whereas vascularity was increased by the overexpression of Pim-3 compared with the parental MiaPaca-2, consistent with our previous report (19).

Folkman (45) first developed a theory regarding tumor angiogenesis in 1971, in which he proposed that a tumor produces its own new vasculature from existing blood vessels. Following the introduction of angiogenesis by Folkman in 1971, numerous studies have indicated that tumor cells overexpress a variety of angiogenic genes and secrete various angiogenic factors, including VEGF, PDGF, FGF, EGF and MMP, which recruit vascular ECs into tumor tissues and induce potent angiogenesis. We detected that Pim-3 overexpression increased VEGF content consistent with our previous results (19). Moreover, a loss of Pim-3 kinase activity significantly decreased EGF and FGF mRNA, but not MMP-2 and MMP-9 expression, whereas Pim-3 overexpression markedly increased EGF and FGF expression compared with parental cells. Consequently, the EGF- and FGF-mediated signals may account for neovascularization and subsequently promote tumor growth.

Tumor-associated fibroblasts can produce HGF and are presumed to be crucial in tumor progression (46). We also observed the decreasing mRNA expression of HGF in the intratumoral tissues expressing an inactive Pim-3 kinase. This may mirror the fact that fibroblasts participate in pancreatic carcinogenesis.

Antiangiogenic therapy has shown promise as a treatment for several cancers, such as colon cancer and non-small cell lung cancer $(43,44,47)$. However, the antitumor effects of current angiostatic drugs are short-lived in most patients. Moreover, the overall survival rates for most cancer patients are not significantly prolonged $(48,49)$. Hence, there is a need for other tumor-selective proangiogenic molecules that can be used in combination with or without conventional antiangiogenic drugs. The genetic deficiency of Pim-3 gene does not result in apparent changes in phenotypes, suggesting that Pim-3 may be physiologically dispensable. Unlike other survival kinases, such as the Akt kinases, Pim kinases are not localized downstream of the insulin receptor signaling pathway and, therefore, the inhibition of Pim kinases has few effects on insulin receptor pathway. Thus, Pim-3 would be a preferred target molecule for the development of anticancer drugs against solid tumor angiogenesis, in which Pim-3 is aberrantly expressed. Thus, targeting Pim-3 may play a dual role in halting tumor progression, by promoting tumor cell death and blocking angiogenesis.

\section{Acknowledgements}

The authors would like to express their sincere gratitude to Professor Naofumi Mukaida (Cancer Research Institute, Kanazawa University) for his critical comments on the manuscript. The authors gratefully acknowledge grant support from The National Science Foundation of China (NSFC) (30973476, 812727), the Shanghai Pujiang Program (KW201028464), the Fudan University '985 Project' Phase III Cancer Research Projects II (985III-YFX0102), and the Shanghai Committee of Science and Technology (12DZ2260100).

\section{References}

1. Siegel R, Naishadham D and Jemal A: Cancer statistics, 2013. CA Cancer J Clin 63: 11-30, 2013.

2. Winter JM, Cameron JL, Campbell KA, et al: 1423 pancreaticoduodenectomies for pancreatic cancer: a single-institution experience. J Gastrointest Surg 10: 1199-1211, 2006.

3. Wang Z, Li Y, Ahmad A, et al: Pancreatic cancer: understanding and overcoming chemoresistance. Nat Rev Gastroenterol Hepatol 8: 27-33, 2011.

4. Chu D, Kohlmann W and Adler DG: Identification and screening of individuals at increased risk for pancreatic cancer with emphasis on known environmental and genetic factors and hereditary syndromes. JOP 11: 203-212, 2010.

5. Li D, Xie K, Wolff R and Abbruzzese JL: Pancreatic cancer. Lancet 363: 1049-1057, 2004

6. Vincent A, Herman J, Schulick R, Hruban RH and Goggins M: Pancreatic cancer. Lancet 378: 607-620, 2011.

7. Zavoral M, Minarikova P, Zavada F, Salek C and Minarik M: Molecular biology of pancreatic cancer. World J Gastroenterol 17: 2897-2908, 2011.

8. Li YY, Popivanova BK, Nagai Y, Ishikura H, Fujii C and Mukaida N: Pim-3, a proto-oncogene with serine/threonine kinase activity, is aberrantly expressed in human pancreatic cancer and phosphorylates bad to block bad-mediated apoptosis in human pancreatic cancer cell lines. Cancer Res 66: 6741-6747, 2006.

9. Feldman JD, Vician L, Crispino M, et al: KID-1, a protein kinase induced by depolarization in brain. J Biol Chem 273: 16535-16543, 1998.

10. Deneen B, Welford SM, Ho T, Hernandez F, Kurland I and Denny CT: PIM3 proto-oncogene kinase is a common transcriptional target of divergent EWS/ETS oncoproteins. Mol Cell Biol 23: 3897-3908, 2003.

11. Fujii C, Nakamoto Y, Lu P, et al: Aberrant expression of serine/ threonine kinase Pim-3 in hepatocellular carcinoma development and its role in the proliferation of human hepatoma cell lines. Int J Cancer 114: 209-218, 2005.

12. Popivanova BK, Li YY, Zheng H, et al: Proto-oncogene, Pim-3 with serine/threonine kinase activity, is aberrantly expressed in human colon cancer cells and can prevent Bad-mediated apoptosis. Cancer Sci 98: 321-328, 2007. 
13. Zheng HC, Tsuneyama K, Takahashi H, et al: Aberrant Pim-3 expression is involved in gastric adenoma-adenocarcinoma sequence and cancer progression. J Cancer Res Clin Oncol 134: 481-488, 2008

14. Wu Y, Wang YY, Nakamoto Y, et al: Accelerated hepatocellular carcinoma development in mice expressing the Pim-3 transgene selectively in the liver. Oncogene 29: 2228-2237, 2010.

15. Zhang F, Liu B, Wang Z, et al: A novel regulatory mechanism of Pim-3 kinase stability and its involvement in pancreatic cancer progression. Mol Cancer Res 11: 1508-1520, 2013.

16. Yunis AA, Arimura GK and Russin DJ: Human pancreatic carcinoma (MIA PaCa-2) in continuous culture: sensitivity to asparaginase. Int J Cancer 19: 128-135, 1977.

17. Yano T, Ishikura $\mathrm{H}$, Kato $\mathrm{H}$, et al: Vaccination effect of interleukin-6-producing pancreatic cancer cells in nude mice: a model of tumor prevention and treatment in immune-compromised patients. Jpn J Cancer Res 92: 83-87, 2001.

18. Wang YY, Taniguchi T, Baba T, Li YY, Ishibashi $\mathrm{H}$ and Mukaida N: Identification of a phenanthrene derivative as a potent anticancer drug with Pim kinase inhibitory activity. Cancer Sci 103: 107-115, 2012.

19. Wang C, Li HY, Liu B, Huang S, Wu L and Li YY: Pim-3 promotes the growth of human pancreatic cancer in the orthotopic nude mouse model through vascular endothelium growth factor. J Surg Res 185: 595-604, 2013

20. Xu D, Cobb MG, Gavilano L, et al: Inhibition of oncogenic Pim-3 kinase modulates transformed growth and chemosensitizes pancreatic cancer cells to gemcitabine. Cancer Biol Ther 14 492-501, 2013

21. Qian KC, Wang L, Hickey ER, et al: Structural basis of constitutive activity and a unique nucleotide binding mode of human Pim-1 kinase. J Biol Chem 280: 6130-6137, 2005.

22. Yang XY, Ren CP, Wang L, et al: Identification of differentially expressed genes in metastatic and non-metastatic nasopharyngeal carcinoma cells by suppression subtractive hybridization. Cell Oncol 27: 215-223, 2005.

23. Byers LA, Sen B, Saigal B, et al: Reciprocal regulation of c-Src and STAT3 in non-small cell lung cancer. Clin Cancer Res 15: 6852-6861, 2009.

24. He M and Young CY: New approaches to target the androgen receptor and STAT3 for prostate cancer treatments. Mini Rev Med Chem 9: 395-400, 2009.

25. Kim DY, Cha ST, Ahn DH, et al: STAT3 expression in gastric cancer indicates a poor prognosis. J Gastroenterol Hepatol 24: 646-651, 2009.

26. Scholz A, Heinze S, Detjen KM, et al: Activated signal transducer and activator of transcription 3 (STAT3) supports the malignant phenotype of human pancreatic cancer. Gastroenterology 125 : 891-905, 2003

27. Hirano T, Ishihara K and Hibi M: Roles of STAT3 in mediating the cell growth, differentiation and survival signals relayed through the IL-6 family of cytokine receptors. Oncogene 19 2548-2556, 2000.

28. Shirogane T, Fukada T, Muller JM, Shima DT, Hibi M and Hirano T: Synergistic roles for Pim-1 and c-Myc in STAT3mediated cell cycle progression and antiapoptosis. Immunity 11: 709-719, 1999

29. Aksoy I, Sakabedoyan C, Bourillot PY, et al: Self-renewal of murine embryonic stem cells is supported by the serine/threonine kinases Pim-1 and Pim-3. Stem Cells 25: 2996-3004, 2007.

30. Li YY, Wu Y, Tsuneyama K, Baba T and Mukaida N: Essential contribution of Ets-1 to constitutive Pim-3 expression in human pancreatic cancer cells. Cancer Sci 100: 396-404, 2009.
31. Mackenzie GG, Huang L, Alston N, et al: Targeting mitochondrial STAT3 with the novel phospho-valproic acid (MDC-1112) inhibits pancreatic cancer growth in mice. PLoS One 8: e61532, 2013.

32. You W, Tang Q, Zhang C, et al: IL-26 promotes the proliferation and survival of human gastric cancer cells by regulating the balance of STAT1 and STAT3 activation. PLoS One 8: e63588, 2013.

33. Thoennissen NH, Iwanski GB, Doan NB, et al: Cucurbitacin B induces apoptosis by inhibition of the JAK/STAT pathway and potentiates antiproliferative effects of gemcitabine on pancreatic cancer cells. Cancer Res 69: 5876-5884, 2009.

34. Chang M, Kanwar N, Feng E, et al: PIM kinase inhibitors downregulate STAT3(Try705) phosphorylation. Mol Cancer Ther 9: 2478-2487, 2010

35. Talbot DC, Ranson M, Davies J, et al: Tumor survivin is downregulated by the antisense oligonucleotide LY2181308: a proof-of-concept, first-in-human dose study. Clin Cancer Res 16: 6150-6158, 2010

36. Sah NK, Khan Z, Khan GJ and Bisen PS: Structural, functional and therapeutic biology of survivin. Cancer Lett 244: 164-171, 2006.

37. Liu BB and Wang WH: Survivin and pancreatic cancer. World J Clin Oncol 2: 164-168, 2011.

38. Diaz N, Minton S, Cox C, et al: Activation of stat 3 in primary tumors from high-risk breast cancer patients is associated with elevated levels of activated SRC and survivin expression. Clin Cancer Res 12: 20-28, 2006.

39. Kanwar JR, Kamalapuram SK and Kanwar RK: Targeting survivin in cancer: the cell-signalling perspective. Drug Discov Today 16: 485-494, 2011.

40. O'Connor DS, Grossman D, Plescia J, et al: Regulation of apoptosis at cell division by $\mathrm{p} 34 \mathrm{cdc} 2$ phosphorylation of survivin. Proc Natl Acad Sci USA 97: 13103-13107, 2000.

41. Wheatley SP and McNeish IA: Survivin: a protein with dual roles in mitosis and apoptosis. Int Rev Cytol 247: 35-88, 2005.

42. Zhang Y, Park TS and Gidday JM: Hypoxic preconditioning protects human brain endothelium from ischemic apoptosis by Akt-dependent survivin activation. Am J Physiol Heart Circ Physiol 292: H2573-H2581, 2007.

43. Hanahan D and Weinberg RA: Hallmarks of cancer: the next generation. Cell 144: 646-674, 2011.

44. Cao Z, Shang B, Zhang G, et al: Tumor cell-mediated neovascularization and lymphangiogenesis contrive tumor progression and cancer metastasis. Biochim Biophys Acta 1836: 273-286, 2013.

45. Folkman J: Tumor angiogenesis: therapeutic implications. $\mathrm{N}$ Engl J Med 285: 1182-1186, 1971.

46. Kalluri R and Zeisberg M: Fibroblasts in cancer. Nat Rev Cancer 6: 392-401, 2006.

47. Carmeliet $P$ and Jain RK: Molecular mechanisms and clinical applications of angiogenesis. Nature 473: 298-307, 2011.

48. Johannessen TC, Wagner M, Straume O, Bjerkvig R and Eikesdal HP: Tumor vasculature: the Achilles' heel of cancer? Expert Opin Ther Targets 17: 7-20, 2013.

49. De Bock K, Mazzone M and Carmeliet P: Antiangiogenic therapy, hypoxia, and metastasis: risky liaisons, or not? Nat Rev Clin Oncol 8: 393-404, 2011. 